\title{
ADJUSTING TWO-DIMENSIONAL VELOCITY DATA TO OBEY CONTINUITY
}

\author{
By L. A. RASMUSSEN
}

(U.S. Geological Survey, Tacoma, Washington 98402, U.S.A.)

\begin{abstract}
An algorithm is developed for adjusting glacier surface-velocity vectors, given on the nodes of a square grid, so that they obey a central-difference approximation of the continuity equation. Also required on the grid nodes are the glacier thickness, the ratio of the surface-velocity to the average velocity in the column, and the difference between the mass balance and the thickness change. All these other variables are assumed to be known exactly, and only the surfacevelocity field is adjusted. The result is optimum in the sense that the magnitude of the adjustment is minimized. Either the relative or the absolute adjustment can be minimized, depending on how weights are specified. No restriction is placed on the shape of the solution region, and no boundary condition is required. The algorithm is not iterative. The algorithm first forms a parallel flow field that satisfies the continuity equation, and then uses a stream function to add a divergenceless field to it. The stream function that leads to the minimum velocity adjustment is obtained as four independent, interlacing solutions covering the solution region. For each of the four, a well-conditioned, sparse-matrix system of simultaneous linear equations is solved. A compact, sub-optimum, well-behaved iterative procedure is also developed for transforming part of the velocity adjustment into an adjustment of the thickness field.
\end{abstract}

RÉSUMÉ. Ajustement d'un champ de vitesses bidimensionnel observe afin de respecter l'équation de continuité. On développe un algorithme qui permet d'ajuster des vecteurs vitesse superficielle donnés aux noeuds d'une grille carrée de manière à ce qu'ils obéissent à un schéma aux différences centrées de l'équation de continuité. Pour ce faire, il faut également connaitre aux noeuds de la grille l'épaisseur du glacier, le rapport entre la vitesse en surface et la vitesse moyenne sur une verticale ainsi que la différence entre bilan de masse et variation d'épaisseur. Toutes ces variables sont supposées parfaitement connues et seul le champ de vitesse superficielle est ajusté. L'optimisation est réalisée en minimisant la valeur de l'ajustement. On peut, en spécifiant les poids respectifs, minimiser les valeurs d'ajustements relatives ou absolues. La forme de la région soumise au calcul peut être quelconque, aucune condition aux limites n'est nécessaire et l'algorithme

\section{INTRODUCTION}

For some ice-mass configurations - confluences of tributaries, valley glaciers with high curvature of the centerline, ice sheets - the explicit treatment of transverse variations of the flow may yield better results than representing them in parameterized form in a one-dimensional model. The one-dimensional parameterization is effected by introducing shape factors, which have a partly theoretical, partly empirical basis. A two-dimensional flow model - by which is meant that there are two independent horizontal space coordinates $(x, y)$ - avoids the need for shape factors to parameterize the transverse variations.

Whatever a model's dimensionality, its application to an actual ice mass must begin by obtaining a set of n'est pas itératif. L'algorithme construit d'abord un champ de vitesses parallèles satisfaisant l'équation de continuité puis, utilisant une fonction de courant, lui ajoute un champ à divergence nulle. La fonction de courant correspondant à l'ajustement minimal des vitesses est obtenue comme combinaison de quatre solutions indépendantes couvrant la région. Pour chacune des quatre solutions, on résoud un système linéaire dont la matrice est creuse et bien conditionnée. Une procédure itérative compacte, sub optimale, stable et convergente est également développée afin de transformer une partie de l'ajustement cinématique en un ajustement des épaisseurs.

ZUSAMMENFASSUNG. Einpassung zweidimensionaler Geschwindigkeitsdaten in die Kontinuitätsgleichung. Es wird ein Rechenverfahren zur Einpassung von oberflächlichen Geschwindigkeitsvektoren in den Schnittpunkten eines Quadratnetzes auf eine zentral-differentielle Annäherung der Kontinuitätsgleichung entwickelt. In den Netzpunkten werden weiter benötigt: die Gletscherdicke, das Verhältnis zwischen der Oberflăchengeschwindigkeit und der mittleren Geschwindigkeit in der Säule und die Differenz zwischen der Massenbilanz und der Dickenänderung. Diese Parameter werden als fehlerfreie Grössen betrachtet, so dass sich die Einpassung nur auf das oberflächliche Geschwindigkeitsfeld erstreckt. Das Ergebnis ist insofern optimal, als die Einpassung minimiert wird. Dies kann entweder mit der relativen oder der absoluten Ausgleichung geschehen - je nach der Gewichtsfestsetzung. Die Form des Lősungsbereiches unterliegt keinen Einschränkungen; eine Randbedingung wird nicht benötigt. Der Algorithmus ist nicht iterativ. Der Algorithmus erzeugt zuerst ein paralleles Strömungsfeld, das die Kontinuitätsgleichung erfüllt, und zieht dann eine Strömungsfunktion zur Addition eines divergenzfreien Feldes heran. Die Strömungsfunktion, die zur Ausgleichung der Geschwindigkeiten führt, wird aus 4 unabhängigen, verknüpften Lösungen, die den Lösungsbereich überdecken, gewonnen. Für jede der 4 ist ein gut konditioniertes Matrizen-System für gleichzeitige lineare Gleichungen zu lösen. Ein kompaktes, nicht ganz optimales, aber günstiges Iterationsverfahren zur Transformation eines Teiles der Geschwindigkeitsausgleichung in eine Ausgleichung des Dickenfeldes wird ebenfalls vorgeführt.

initial conditions that are consistent with respect to the particular form of the dynamic equations employed by the model, including the continuity equation, and that are faithful, within the bounds of observation error, to the available field data. If they are not consistent, the model would rapidly redistribute the mass, not as a realistic projection of the future behavior of the ice mass but, artificially, to reconcile the inconsistencies. Slight errors in the velocity data can be amplified into spuriously large changes in glacier thickness.

If a particular form of the flow law is not assumed, the data must still be consistent with respect to the continuity equation. Such a data set, obeying continuity and faithful to the field data, would be useful in estimating the numerical values of the flow-law parameters and the amount of sliding motion. 
Adjusting data to be consistent is much more difficult in two dimensions than it is in one. Velocity adjustment in one dimension is easy: flow enters an element at one side, it leaves at the other side, and the difference between the two fluxes equals the integral over the element of the difference between the mass balance and thickness change. Beginning with an assumed or estimated flux value at one end of a one-dimensional domain, a simple integration gives values directly at all the other nodes. In two dimensions, however, it is not always obvious whether any particular side of an element carries inflow or outflow. If the flow is roughly parallel to one axis of two-dimensional grid, then clearly the up-stream side has inflow, and the down-stream side has outflow, but the lesser flow across the other two sides may have either sign. Or, if the flow is diagonal to a two-dimensional grid, so that the sign of the flux across each side is obvious, there is still not a one-to-one correspondence between the flux across one side and the flux across another side; this one-to-one correspondence is what makes the one-dimensional adjustment algorithm so simple, and so compact.

Also, a complete solution, in which all the variables are adjusted, is more difficult than a partial solution, in which only one is adjusted and the others are taken to be exact. The velocity and thickness appear non-linearly, as a product, in the continuity equation. Different adjustment criteria may be appropriate for different variables. The number of values to be adjusted is greater in a complete solution, which substantially increases the amount of computation.

Developed here is an algorithm that:

(a) adjusts only the velocity and assumes the other variables to be known, including the glacier thickness and the ratio of the surface velocity to the average velocity in the vertical column;

(b) requires values of all variables on a square grid, including initial estimates of the velocity; but requires no special conditions on the boundary;

(c) produces velocity values exactly satisfying a central-difference form of the continuity equation;

(d) uses a non-iterative procedure that minimizes the magnitude of the adjustment of the original velocity values, either the absolute adjustment or the relative adjustment; and

(e) is applicable to an arbitrarily shaped solution region.

Also developed is a compact, sub-optimum, well-behaved iterative procedure for transforming part of the velocity adjustment into a thickness adjustment.

\section{CONTINUITY EQUATION}

The horizontal $\xi$-axis is taken to be positive in the direction of the flow, and the $z$-axis is taken to be vertical, positive upwards (Fig. 1). The horizontal component $s$ of the glacier velocity at the surface $Z$ is assumed to be the sum of a sliding part $s_{\mathrm{B}}$, which is constant with $z$, and a part $s_{\mathrm{D}}$ due to deformation under simple shear that varies according to a power law in $z$ :

$$
s_{\mathrm{D}}(z)=s_{\mathrm{D}}-A \int_{Z}^{Z}[\rho g(Z-z) \sin \alpha \cos \alpha]^{n} \mathrm{~d} z,
$$

in which $A$ and $n$ are the flow-law parameters, $\rho$ is the ice density, $g$ is the acceleration due to gravity, and the surface slope $\alpha$ is positive if the surface slopes down in the direction of flow. The bed slope is assumed not to

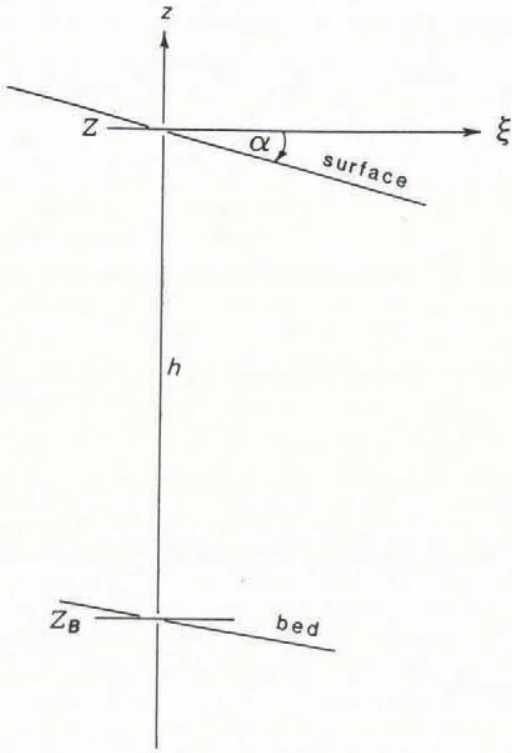

Fig. 1. Vertical section through the glacier thickness. The horizontal coordinate $\xi$ is in the direction of the glacier flow.

differ markedly from the surface slope and it is also assumed that there are no gradients of longitudinal stress or basal or sidewall drag. The value at the surface $z=Z$ is denoted $s$ and similarly for other variables, but below the surface $\left(Z_{\mathrm{B}} \leqslant z<Z\right)$ it is denoted $s_{\mathrm{D}}(z)$. If $s_{\mathrm{D}}(z)=0$ at the glacier bed $z=Z_{\mathrm{B}}$, then on performing the integration through the glacier thickness, $h=Z-Z_{\mathrm{B}}$,

$$
{ }^{s} \mathrm{D}^{(z)}=\left[1-\left[\frac{z-z}{h}\right)^{n+1}\right] s_{\mathrm{D}} .
$$

The area flux $q_{\mathrm{D}}$ (volume flux per unit width) from the deformational part is obtained by another vertical integration:

$$
q_{\mathrm{D}}=\int_{Z_{\mathrm{B}}}^{Z} s_{\mathrm{D}}(z) \mathrm{d} z=\frac{n+1}{n+2} s_{\mathrm{D}^{h}},
$$

so that the total flux is

$$
q=q_{\mathrm{D}}+q_{\mathrm{B}}=\left[\frac{n+1}{n+2} s_{\mathrm{D}}+s_{\mathrm{B}}\right] h=y s h,
$$

which, when the factor $y$ is introduced, is expressed in terms of the thickness and the surface velocity. From Equation (4), since $s=s_{\mathrm{D}}+s_{\mathrm{B}}$,

$$
y=\frac{n+1}{n+2} \cdot \frac{s_{\mathrm{D}}}{s}+\frac{s_{\mathrm{B}}}{s} .
$$

A characteristic thickness $\bar{h} \equiv y h$ may be introduced; it product $\bar{h} s$ with the surface velocity gives the same flux $q$ as the integral of the actual velocity profile through the actual thickness.

The foregoing is an idealized formulation, based on several simplifying physical assumptions, for determining $y$. A more precise and complete formulation would be appropriate only if sufficient data existed for applying it. What follows, however, as stated essentially by point (a) above, pertains to a data set in which $y$ is specified, by whatever means may be used. Moreover, it is the product $y h$ that is used in conjunction with the surface velocity, and in actual practice difficulties may arise in determining $h$ accurately as well as in determining $y$. 


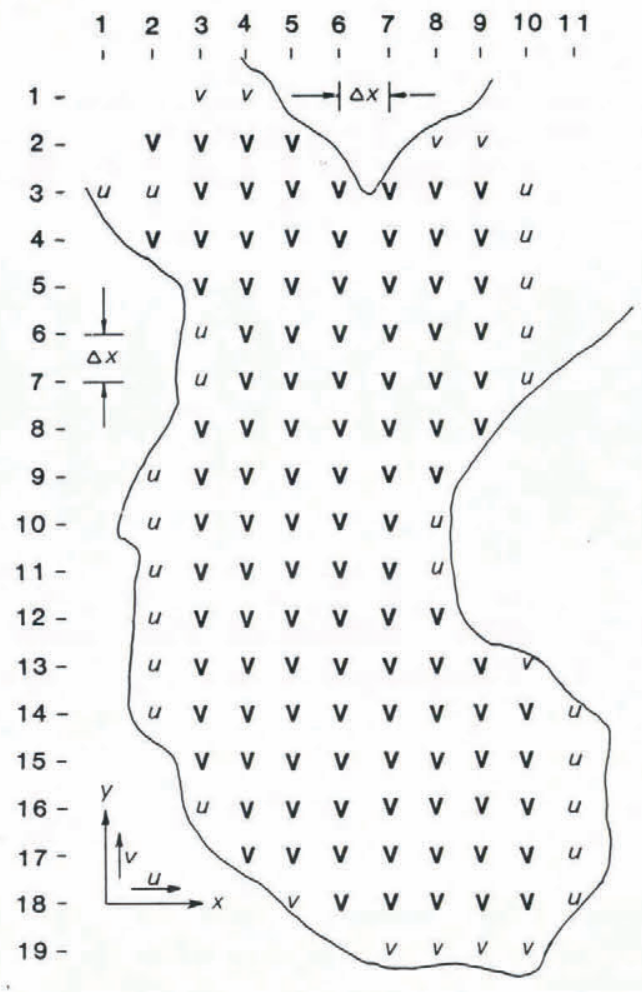

Fig. 2. Horizontal coordinate system, grid indices, and solution region of a hypothetical problem. The symbols $u$. $v$, and $\mathcal{L}$ indicate whether one, the other, or both components are to be adjusted.

When the surface velocity $\mathbf{V}$ is referred to an arbitrary horizontal coordinate system, with components $u$ in the $x$-direction and $v$ in the $y$-direction, the speed is $s=\left(u^{2}+v^{2}\right)^{\frac{1}{2}}$. Considering an infinitesimal element in the $x, y$ plane, and assuming the density $\rho$ to be constant, the conservation of mass may be expressed in the form of the continuity equation as

$$
\dot{b}-\dot{h}=\nabla \cdot Q=\nabla \cdot \underline{\underline{W}} \equiv \frac{\partial}{\partial x}(u \bar{h})+\frac{\partial}{\partial y}(\overline{v h}),
$$

in which $\dot{h}=\check{Z}$ is the time rate of change of the thickness, assuming $\dot{Z}_{\mathrm{B}}=0$, and $\dot{b}$ is the net ice balance at both the top and the bottom of the column, as well as from exchange with englacial liquid-phase water. Equation (6) may be approximated by central differences on a square grid with spacing $\nabla x$ (Fig. 2) as

$$
\begin{aligned}
(u \bar{h})_{i j+1} & -(u \bar{h})_{i j-1}+(v \bar{h})_{i-1} j-(v \bar{h})_{i+1} j= \\
= & 2 \Delta x(\dot{b}-h)_{i j} \equiv F_{i j} .
\end{aligned}
$$

Slight errors in the velocity data - one source of which is the interpolation of field data to the grid nodes - can produce large residuals in Equation (7). An error in one of the velocity components may be small relative to $s$, but it will generally be large relative to $b-h$; unless $\Delta x$ is very large compared with $h$, this will produce a large residual relative to $F$.

\section{THE ALGORITHM}

The problem may be posed formally as finding the velocity field $\mathbf{V}$ that satisfies continuity and that minimizes some measure of its difference from the original velocity field $\mathbf{V}_{0}$. A least-squares form is used here for this measure, termed the adjustment magnitude,

$E=\sum_{i j} \lambda_{i j}\left\{\left[u_{i j}-\left(u_{0}\right)_{i j}\right]^{2}+\left[v_{i j}-\left(v_{0}\right)_{i j}\right]^{2}\right\}$

which can be used to represent either the absolute adjustment, if $\lambda_{i j}=1$, or the relative adjustment, if $\lambda_{i j}=\left(s_{0}\right)_{i j}^{-2}$

If $\bigvee_{1}$ and $\mathbf{V}_{2}$ are any two fields satisfying Equation (6), then

$$
\nabla \cdot\left(\mathbf{V}_{2}-\mathbf{V}_{1}\right) \bar{h}=\nabla \cdot \mathbf{V}_{2} \bar{h}-\nabla \cdot \underline{y}_{1} \bar{h}=0
$$

Therefore, given any field $\mathrm{V}_{1}$ satisfying continuity, the problem is transformed into that of finding the field $\mathbf{V}_{2}$ that satisfies Equation (9) and that minimizes $E$. Because any vector that obeys a stream function is identically divergenceless, if the field $\left(\mathbf{V}_{2}-\mathbf{V}_{1}\right) \bar{h}$ is defined by

$$
\left.\begin{array}{l}
\left(u_{2}-u_{1}\right) \bar{h}=-\partial \psi / \partial y, \\
\left(v_{2}-v_{1}\right) \bar{h}=\partial \psi / \partial x,
\end{array}\right\}
$$

in which the scalar field $\psi$ is the stream function, then Equation (9) is satisfied. If central-difference approximations

$$
\left.\begin{array}{l}
{\left[\left(u_{2}-u_{1}\right) \bar{h}_{i j}=\left(\Psi_{i+1} j-\Psi_{i-1} j\right) /(2 \Delta x),\right.} \\
{\left[\left(v_{2}-v_{1}\right) \bar{h}_{i j}=\left(\Psi_{i j+1}-\Psi_{i j-1}\right) /(2 \Delta x)\right.}
\end{array}\right\}
$$

are used for the $\psi$-derivatives, then the central-difference approximation of Equation (9) is also exactly satisfied.

On substituting for $\left(u_{2}\right)_{i j}$ and $\left(v_{2}\right)_{i j}$ from Equations (11), and introducing them into Equation (8),

$$
\begin{aligned}
E & =\sum_{i j} \lambda_{i j}\left\{\left[u_{1}-u_{0}\right)_{i j}+\left(\Psi_{i+1} j-\Psi_{i-1} j\right) / H_{i j}\right]^{2}+ \\
& \left.+\left[\left(v_{1}-v_{0}\right)_{i j}+\left(\Psi_{i j+1}-\Psi_{i j-1}\right) / H_{i j}\right]^{2}\right\},
\end{aligned}
$$

in which $H_{i j}=2 \bar{h}_{i j} \Delta x$. Setting $\partial E / \partial \Psi_{i j}$ to zero for all $\Psi_{i j}$ leads to the system of simultaneous linear equations:

$$
\begin{gathered}
\left(c_{i j+1}+c_{i j-1}+c_{i+1} j+c_{i-1} j\right) \Psi_{i j}- \\
-c_{i j+1} \Psi_{i j+2}-c_{i j-1} \Psi_{i j-2}-c_{i j+1} j \Psi_{i+2} j-c_{i-1} j \Psi_{i-2} j \\
=\left[\left(v_{1}-v_{0}\right) d\right]_{i j+1}-\left[\left(v_{1}-v_{0}\right) d\right]_{i j-1}+\left[\left(u_{1}-u_{0}\right) d\right]_{i+1} j-\left[\left(u_{1}-u_{0}\right) d\right]_{i-1} j
\end{gathered}
$$


TABLE I. FOUR INDEPENDENT, INTERLACING SUBSETS OF THE SOLUTION REGION. INDICATED FOR EACH SUBSET ARE THE ROW AND COLUMN COMBINATIONS FOR WHICH THE $\psi$-VALUES ARE COMPUTED (FROM EQUATIONS 13), ON WHICH THE $u_{2}$-VALUES AND $v_{2}$-VALUES ARE THEN DETERMINED (FROM EQUATIONS 11), AND ON WHICH THE $F$-VALUES ARE USED TO GET THE $u_{1}$-VALUES AND $v_{1}$-VALUES (FROM EQUATIONS 14)

\begin{tabular}{|c|c|c|c|c|c|c|c|c|}
\hline \multirow[b]{2}{*}{ Subset } & \multicolumn{2}{|c|}{$\Psi$} & \multicolumn{2}{|c|}{$u$} & \multicolumn{2}{|c|}{$v$} & \multicolumn{2}{|c|}{$f$} \\
\hline & rows & columns & rows & columns & rows & columns & rows & columns \\
\hline I & even & even & odd & even & even & odd & odd & odd \\
\hline II & even & odd & odd & odd & even & even & odd & even \\
\hline III & odd & even & even & even & odd & odd & even & odd \\
\hline IV & odd & odd & even & odd & odd & even & even & even \\
\hline
\end{tabular}

in which $c_{i j}=d_{i j} / H_{i j}$ and $d_{i j}=\lambda_{i j} / \bar{h}_{i j}$. Because the $\Psi$-subscript increments are all \pm 2 , the determination of the $\psi_{i j}$ requires solving four separate systems of linear equations, one on each of the four independent, interlacing subsets (Table I, Fig. 3) of the grid nodes in the solution region. Each of the four linear systems is singly underdetermined - the $\psi$-differences are unaffected if a constant is added to all the $\psi_{i j}$ - which can be remedied by arbitrarily setting one of the $\psi_{i j}$ to zero, say, and solving for the others. The matrix formed from the left-hand side of Equation (13) is sparse and diagonally dominant; each row contains the diagonal element and from two to four off-diagonal elements, depending on how many $u$ or $v$ values in the solution region surround $\psi_{i j}$ :

Because the $\psi$-field has the same number of degrees of freedom as the $\mathbf{V}_{\text {}}$ field which it produces, a globally optimum solution occurs. The number of degrees of freedom in the $V_{2}$ field is the number of elements less the number of points at which Equation (7) is applied. The number of degrees of freedom in the $\psi$-field is one less than the number of its elements. Through Equations

\begin{tabular}{|c|c|c|c|c|}
\hline & odd & even & odd & even \\
\hline odd & $\frac{u \mid F}{v \mid \psi}$ & $\frac{\left.F\right|_{u}}{\psi_{v}}$ & $\frac{u \mid F}{v \mid \psi}$ & $\frac{F \psi_{u}}{\psi \psi_{v}}$ \\
\hline even & $\frac{\psi_{v}}{F l_{u}}$ & $\frac{v \psi \psi}{u F}$ & $\frac{\psi}{\left.F\right|_{u}}$ & $\frac{v \psi}{u \mid F}$ \\
\hline odd & $\frac{u \mid F}{v \mid \psi}$ & $\frac{F \psi_{u}}{\psi_{v}}$ & $\frac{u \mid F}{v \mid \psi}$ & $\frac{F \mid u}{\psi \|_{v}}$ \\
\hline even & $\frac{\psi_{v}}{F l_{u}}$ & $\frac{v \psi}{u \psi F}$ & $\frac{\psi_{l_{v}}}{\text { Flu }}$ & $\frac{v \psi \psi}{u F}$ \\
\hline odd & $\frac{u \mid F}{v \psi \psi}$ & $\frac{F \psi_{u}}{\psi \psi_{v}}$ & $\frac{u \mid F}{V \mid \psi}$ & $\frac{\left.F\right|_{u}}{\psi_{V}}$ \\
\hline
\end{tabular}

Subset key $\frac{\text { IIII }}{\text { पII }}$

Fig. 3. Patterns of the four independent, interlacing subsets of the solution region, illustrating Table I. The key at the bottom shows to which subset each of the four corners of the pattern at each grid node pertains. For example, subset IV gives $u$-values on the even-row, odd-column nodes and it gives $v$-values on the odd-row, even-column nodes.
(11), the ${\underset{\sim}{2}}_{2}$ field is produced in terms of a $\mathbf{V}_{1}$ field assumed to satisfy Equation (7). For instance, a parallel-flow solution for $\mathbf{V}_{1}$ is

$v_{i j}=0$

$u_{i j}=\left\{\begin{array}{lc}0 & \left(j \geqslant 2 \text { or } h_{i j}=0\right) \\ \left(F_{i j-1}+u_{i j-2} \bar{h}_{i j-2}\right) / \bar{h}_{i j} & \left(j \geqslant 3 \text { and } \bar{h}_{i j}>0\right)\end{array}\right\}$

Although a mathematical proof cannot be adduced here, numerical experimentation revealed that the resulting $\mathbf{V}_{2}$ field is independent of the $V_{1}$ field used. Exactly the same $\mathbf{Y}_{2}$ field was obtained when $\mathbf{V}_{1}$ was obtained from Equations (14) by setting the $u_{i j}=0$ and making the $v_{i j}$ satisf y Equation (7), and also when another $\mathrm{V}_{1}$ better resembling $\mathbf{V}_{0}$ was used with both $u_{i j}$ and $v_{i j}$ generally non-zero. The uniqueness of the solution $\mathbf{Y}_{2}$ is confirmed intuitively by noting that $E$ is a quadratic norm (Equation (8)) and that the constraint is linear (Equation (7)).

The topology of the solution region can be intricate. If the continuity equation is applied at some point $(i, j)$, then $F$ must be present there; $u$ at $(i, j+1)$ and $(i, j-1) ; v$ at $(i-1, j)$ and $(i+1, j)$; and $\psi$ at $(i-1, j+1),(i-1, j-1)$, $(i+1, j+1)$, and $(i+1, j-1)$. Table I gives the patterns for each of the four independent, interlacing subsets of the solution region. Figure 2 shows a hypothetical solution region in terms of the nodes at which $u$ and/or $v$ are to be adjusted; it includes an unusual point $(3,2)$ at which the continuity equation is applied, but at which only the $u$-component is to be adjusted. Figure 4 shows the corresponding subset II in terms of the patterns of $F, u$, $v$, and $\psi$.

\section{ADJUSTING THICKNESS DATA}

Consideration of Equation (7) reveals that if $\bar{h}$ and $\bigvee_{2}$ satisfy it at any point $(i, j)$, then so do $\bar{h} / p$ and $p \mathbf{V}_{2}$ for any $p>0$. This makes it easy to reduce the velocity adjustment $E$ by transforming part of it into an adjustment of the $\bar{h}$ field. The magnitude of the compound adjustment, in which $v$ is an arbitrary constant to scale, the contributions from the two variables,

$$
\begin{gathered}
E^{\prime}=\sum_{i j} \mu_{i j}\left(\bar{h}_{i j} / p_{i j}-\bar{h}_{i j}\right)^{2}+ \\
\left.+v \lambda_{i j}\left\{\left[p_{i j}\left(u_{2}\right)_{i j}-u_{0}\right)_{i j}\right]^{2}+\left[p_{i j}\left(v_{2}\right)_{i j}-\left(v_{0}\right)_{i j}\right]^{2}\right\}
\end{gathered}
$$

may represent either the relative or the absolute magnitude of the velocity adjustment, depending on how 


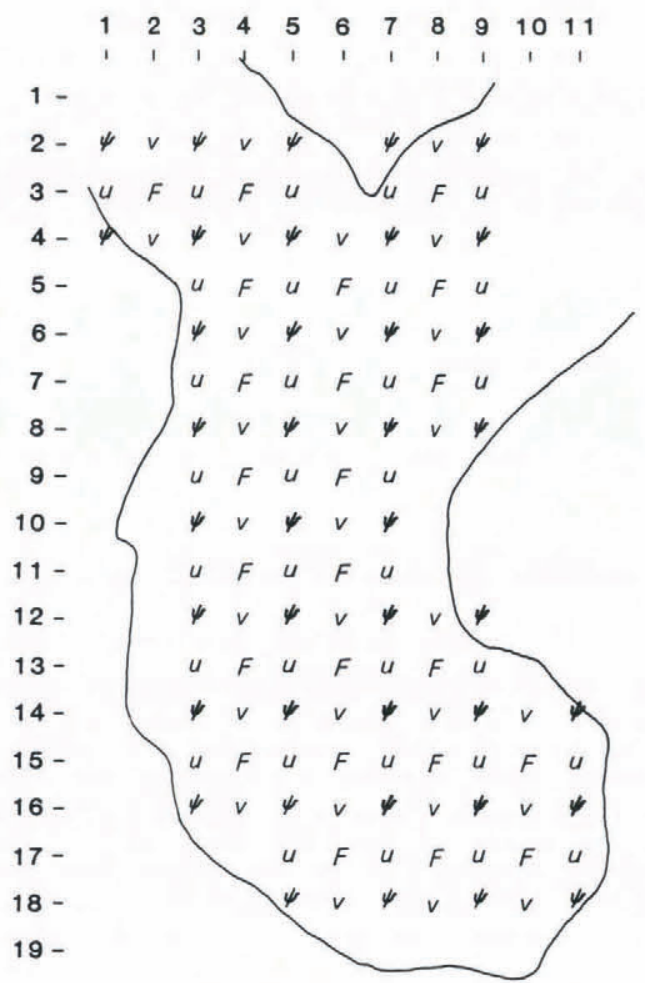

Fig. 4. Subset II of the solution region shown in Figure 2. The continuity equation is applied at nodes indicated by $F$ to adjust components $u$ and $v$ at nodes indicated by those symbols by means of the stream function at nodes indicated by $\Psi$. $\lambda_{i j}$ is assigned, and may represent either the absolute thickness adjustment if $\mu_{i j}=1$, or the relative adjustment if $\mu_{i j}=\bar{h}_{i j}^{-2}$. Because the adjustment at one point is independent of the adjustments at other points, $E^{\prime}$ can be minimized by minimizing the contributions from the individual points. However, this piecewise minimization, first $E$ and then $E^{\prime}$, does not produce as small an $E^{\prime}$ as would occur were $\bar{h}$ and $\mathbf{V}_{2}$ adjusted simultaneously. The contribution at some point is minimized by setting $\partial E_{i j}^{\prime} / \partial p_{i j}=0$, which gives rise to the quartic polynomial (the subscripts having been dropped)

in which

$$
M p^{4}-N p^{3}+p-1=0
$$

$M=v \lambda s_{2}{ }^{2} /\left(\bar{\mu} h^{2}\right)$ and $N=v \lambda\left(u_{0} u_{2}+v_{0} v_{2}\right) /\left(\bar{\mu} h^{2}\right)$. form

The quartic may be rearranged into the convenient

$$
p=\frac{1+N p^{3}}{1+M p^{3}} \equiv \Phi(p),
$$

and may easily be solved iteratively. The function $\Phi(p)$ varies monotonically from $\Phi(0)=1$ and $\mathrm{d} \Phi(0) / \mathrm{d} p=0$, to $\Phi(\infty)=N / M$ and $\mathrm{d} \Phi(\infty) / \mathrm{d} p=0$. A good approximation is

$$
p_{0}=\frac{(1+M)^{3}+N(1+N)^{3}}{(1+M)^{3}+M(1+N)^{3}},
$$

which may suffice in some applications as itself an acceptable value of $p$ or, if not, provides a first approximation for numerically solving Equation (17).

MS. received 6 February 1984 and in revised form 6 November 1984 